\title{
The Impact of the Uighur Medicine Seeds of Nigella Glandulifera Freyn (SNF) on Antitumor and Antioxidant Activity in a S180 and EAC Mouse Tumor Model
}

\author{
Dilinuer Maimaitiyiming ${ }^{\#}$ \\ Comprehensive Cardiac Medicine \\ First Teaching hospital of Xinjiang Medical University \\ Urumqi, Xinjiang, China \\ e-mail: 115149469@qq.com \\ Amanguli" \\ Central Laboratory \\ Xinjiang Medical University \\ Urumqi, Xinjiang, China \\ e-mail:18690893065@163.com
}

\section{Li Yan}

Central Laboratory

Xinjiang Medical University

Urumqi, Xinjiang, China

e-mail: yltension@163.com

$$
\begin{gathered}
\text { Nazuk Kurbantay } \\
\text { Central Laboratory } \\
\text { Xinjiang Medical University } \\
\text { Urumqi, Xinjiang, China } \\
\text { e-mail: 243165806@qq.com } \\
\text { Guizhen Wu } \\
\text { Central Laboratory } \\
\text { Xinjiang Medical University } \\
\text { Urumqi, Xinjiang, China } \\
\text { e-mail: Wuguizhen@126.com } \\
\text { Lianlian Shan } \\
\text { Department of Drug Analysis } \\
\text { Xinjiang Medical University } \\
\text { Urumqi, Xinjiang, China } \\
\text { e-mail: happyshanlian@ sina.com }
\end{gathered}
$$

\author{
Zuhragul Omarniyaz \\ Department of Drug Analysis \\ Xinjiang Medical University \\ Urumqi, Xinjiang, China \\ e-mail: 760272647@qq.com
}

Tao Yang

Department of Drug Analysis

Xinjiang Medical University

Urumqi, Xinjiang, China

e-mail: 690579893@qq.com

Shuge Tian

Central Laboratory

Xinjiang Medical University

Urumqi, Xinjiang, China

e-mail: 675552462@qq.com

\author{
Halmurat Upur \\ Traditional Uighur Medicine and Pharmacology \\ Xinjiang Medical University \\ Urumqi, Xinjiang, China \\ e-mail: halmurat@263.net
}

\author{
Ainiwaer Aikemu* \\ (Corresponding author) \\ Central Laboratory \\ Xinjiang Medical University \\ Urumqi, Xinjiang, China \\ e-mail: ainiwa@sina.com
}

${ }^{\#}$ These authors are contributed equally to this work

\begin{abstract}
Objective: This study was designed to study the antitumor, antioxidant activity and antihypoxia of Uighur medicine Seeds of Nigella glandulifera Freyn (SNF) in the S180 and EAC mice tumor model. Methods: A total of $\mathbf{1 0 0}$ were randomly divided into five groups with 20 animals in each group: control group, model group, Seeds of Nigella glandulifera Freyn ethanol extract (SNFEE) group, Seeds of Nigella glandulifera Freyn water extract (SNFWE) group, cyclophosphamide group. The serum levels of superoxide dismutase (SOD), malonaldehyde (MDA), and glutathione-catalase (GSH-PX) were analyzed, and the mice
\end{abstract}

were also subjected to a hypoxia tolerance test. Their climbing ability was also analyzed. Results: The findings of the study revealed that $\mathrm{SNF}$-treatment leads to an increase in blood serum SOD and GSH-PX levels but a decrease in blood serum MDA levels. Moreover, SNF-treatment enhanced the survival time of mice maintained under hypoxic conditions and improved their mice climbing ability. Conclusion: The results of this study indicate that SNF has obvious antitumor, antihypoxia and antioxidative effects.

Keywords-Uighur medicine; Seeds of Nigella Glandulifera Freyn; antioxidant; antitumor; antihypoxia 


\section{INTRODUCTION}

Seeds of Nigella glandulifera Freyn is widely distributed in the Xinjiang region of China and has long been used in traditional Uighur medicine for the treatment of diseases such as diuretic, brain and kidney supplement, blood circulation improvement, detoxification, galactagogue, menstruation improvement, cough drop, hair blacken and anti-aging [1-5]. Nigella glandulifera Freyn has been studied anti-tumor and immune effects of EAC section indicators of tumor [6]. Study on effect of total saponins from Nigella glandulifera Freyn on inflammatory mediators and ERK/MAPK pathway in stimulated macrophage has been studied [7]. The Investigation of Effective Chemical Groups in Uighur Plant Nigella glandulifera Freyn on Relieving Cough, Expelling Phlegm and Anti-inflammatory has been studied [8]. Nigella glandulifera Freyn's antitussive, expectorant, anti-inflammatory effective parts have been studied [9]. HPLC Fingerprint Spectrum of Nigella glandulifera Freyn has been studied [10]. Uygur hospital to do their preparations immune enhancer for cancer therapy. To sum up the reasons we are ready to study.

\section{MATERIALS AND METHODS}

\section{A. Chemicals and reagents}

Seeds of Nigella glandulifera Freyn (SNF) were purchased in the Minority Hospital of Xinjiang Autonomous Region. Cyclophosphamide was obtained from the HengRui Pharmaceutical Co., Ltd (JiangSu under the State food and Drug Administration, Batch Number: H32020857). The Verapamil Hydrochloride injection preparation was obtained from the Shanghai Hefeng Pharmaceutical Co., Ltd (Batch Number: 090201). The superoxide dismutase (SOD) kit was obtained from Nanjing bio-engineering research institute (Batch Number: 20100420). Similarly, the malonaldehyde (MDA) kit was obtained from the Nanjing bio-engineering research institute (Batch Number: 20100420). The glutathione-catalase (GSH-PX) kit was obtained from the Nanjing bio-engineering research institute (Batch Number: 20100420)

\section{B. Animals and treatment}

Kunming's mice (4-6 weeks old) of the SPF level (body weight $20 \pm 2 \mathrm{~g}$ ) were provided by the Laboratory Animal Center of the XinJiang Medical University. Experiments conducted in China Lanzhou military hospital. The Laboratory Animal Centre of Medicine Animal Care and Use Committee of the China Lanzhou military hospital at Urumqi approved all experimental protocols (No:200905207). The mice were bred under regular laboratory conditions, with a controlled room temperature and a 12/12-hour light-dark cycle with free access to standard rodent chow and water. A total of 100 were randomly divided into five groups with 20 animals in each group: (1) control group, (2) model group, (3) Seeds of Nigella glandulifera Freyn ethanol extract (SNFEE) $(250 \mathrm{mg} / \mathrm{kg})$ group, (4) Seeds of Nigella glandulifera Freyn water extract (SNFWE) $(250 \mathrm{mg} / \mathrm{kg}$ ) group, (5) cyclophosphamide (CY) group. The S180 cells were supplied by the Laboratory Animal Center of Xinjiang Military Hospital and the EAC cells were supplied by the China Center for Type Culture Collection, Wuhan University.

\section{The S180 and EAC transplantation models}

With the exception of the control group, a $0.2 \mathrm{~mL}$ suspension containing $1 \times 10^{7}$ cells/ml of 7 -day-old S180 or EAC cells was transplanted into the right axilla of each mouse. The whole operation was finished in $30 \mathrm{~min}$. The control and model groups were treated with $20 \mathrm{mg} / \mathrm{kg}$ physiological saline, and the SNF and CY groups were treated with their respective doses of drug orally $24 \mathrm{~h}$ after tumor inoculation. The animals' general health statuses were monitored daily for 10 days.

\section{Assessment of tumor weight, thymus, liver, and spleen index}

Twenty-four hours after their last dosage, the mice were killed by cervical dislocation and the thymus, spleen, liver, and solid tumors were excised and weighed. Anticancer activity in vivo was expressed as an inhibitory rate and was calculated using the formula: $[(\mathrm{A}-\mathrm{B}) / \mathrm{A}] \times$ $100 \%$, where $\mathrm{A}$ and $\mathrm{B}$ indicate the mean tumor weights of the model group and experimental groups, respectively. The spleen, liver and thymus were evaluated by the organ index formula: spleen, liver, or thymus weight $(\mathrm{g}) / \mathrm{body}$ weight $(\mathrm{g})$.

\section{E. The effect of SNF treatment on climbing ability in the S180 or EAC tumor-bearing mice}

As previously described, for each cell type 100 mice were randomly divided into five groups. Each group was composed of 20 mice, 10 males and 10 females. This process was repeated for the EAC cells. Twenty-four hours after the cells were implanted the mice were treated with the respective drugs. For the SNF-treated mice, there were two groups who were treated with SNF daily: Seeds of Nigella glandulifera Freyn ethanol extract (SNFEE) $(250 \mathrm{mg} / \mathrm{kg}$ gavage) group; Seeds of Nigella glandulifera Freyn water extract (SNFWE) (250 mg $/ \mathrm{kg}$ gavage) group. Animals in the CY group received a $20 \mathrm{mg} / \mathrm{kg}$ intraperitoneal injection once daily commencing from the second day following cell implantation. In the model group, the mice received a saline gavage once daily. Animals in the control group received no intervention or tumor inoculation. The mice received treatment for 10 days after the last gavage the mice were challenged with the climbing test, in which a length of bamboo $(15 \mathrm{~cm})$ was hung in the air and the mice required to climb from the bottom to the top. Each attempt was timed, and the timer was stopped when the mice fall down. The climbing time for each group was averaged, and the climbing time extension rate was calculated according the formula: (sample group time/ control group time-1) $\times 100 \%$.

\section{F. Mouse blood serum SOD, MDA, and GSH-PX target determination}

As outlined previously, following cell inoculation and 10 days of continuous drug treatment on the 10th day bloody was obtained via an orbital bleed. The samples 
were set aside for $30 \mathrm{~min}$, centrifuged at $3000 \times \mathrm{g}$ for 20 min and, using the respective kits, the blood serum SOD, MDA, or GSH-PX content was analyzed.

\section{G. The effect of SNF treatment in the $S 180$ or EAC tumor-bearing on resistance to hypoxia}

For each cell type, the mice were divided into two groups: normal pressure and hypoxia. For each group, 100 mice were randomly divided into five groups of 20 animals as mentioned in the previous section (four treatment groups and one control group). The mice received treatment $\mathrm{SNF}$, verapamil (Ver; $4 \mathrm{mg} / \mathrm{kg}$ intraperitoneal) or saline treatment continuously for 10 days. Twenty-four hours after the last gavage, the mice were placed into a $250 \mathrm{ml}$ wide-mouth bottle containing sodium lime (one mouse/bottle). Vaseline was used to close the bottle and the time until the mouse died was recorded.

\section{H. Statistical procedures}

Using SPSS 17.0, normality and variance homogeneity were tested. The mean and standard deviation were calculated, followed by single-factor variance analysis and card side examination of the results. The groups were compared by ANOVA and $\mathrm{P}<0.05$ was considered statistically significant.

\section{RESULTS}

\section{A. The influence of mice daily activities}

Following treatment, the mice's behavior, including their drinking, autonomic activities, urination and general appearance were monitored daily. No secretions from the eyes, ears, nose, or mouth were observed. Animals who received SNFEE showed normal activity, mental status, and clean fur. The tumor growth incubation period (TT) was 3 days, and tumor growth velocity (TS) was slower than the model (saline treatment) group. In the group who received SNFWE, normal activity, a good mental state (better than SNFEE group), and clean fur (better than SNFEE group) were noted. In this group, the TT was 3 days; however, TS was slower than in the SNFEE group. In the CY-treated group, normal activity, good mental state (better than SNFEE group), clean fur (better than SNFEE group), and a TT of 4 days and slowest TS of all groups were observed. The model group (saline treatment only) showed decreased activity, enlarged tumor growth, unclean fur and listlessness behavior with a TT of 2 days and TS fastest of all groups were observed.

\section{B. Inhibitory rate on the growth of transplanted tumors in $S 180$ and the organ weight ratio}

The anticancer effects of SNF treatment in the S180-bearing mouse are shown in TABLE I . and TABLE II . Treatment with the CY and SNF resulted in a marked suppression of tumor growth, as observed by the decreased tumor weights. Compared with the model group, the inhibitory rate for SNFEE, SNFWE and CY-treated groups were 42.07, 54.09 and 48.91\%, respectively. The SNFEE groups showed significant inhibition on tumor weight compared with the CY-treated group $(\mathrm{P}<0.05)$. However, the spleen, the liver or thymus index in SNFEE and SNFWE groups did not decrease significantly compared with the model group [TABLE II.]. The results indicated that in vivo SNF treatment did not cause any serious toxic effects on the immune systems of the S180-bearing mice.

TABLE I . ANTITUMOR ACTIVITY OF THE SNF IN MICE TRANSPLANTED S180TUMOR ( $\mathrm{X} \pm$ SD)

\begin{tabular}{ccccccc}
\hline Group & $\begin{array}{c}\text { Dosage } \\
(\mathrm{g} / \mathrm{kg})\end{array}$ & $\begin{array}{c}\text { Tumor weight } \\
(\mathrm{g})\end{array}$ & $\begin{array}{c}\text { Thymus weight } \\
(\mathrm{g})\end{array}$ & $\begin{array}{c}\text { Spleen weight } \\
(\mathrm{g})\end{array}$ & $\begin{array}{c}\text { Liver weight } \\
(\mathrm{g})\end{array}$ & $\begin{array}{c}\text { Body weigh } \\
(\mathrm{g})\end{array}$ \\
\hline control & 0 & $0.000 \pm 0.000$ & $0.106 \pm 0.005$ & $0.098 \pm 0.013$ & $1.204 \pm 0.028$ & $29.770 \pm 0.401$ \\
model & 0 & $0.599 \pm 0.043$ & $0.105 \pm 0.11$ & $0.113 \pm 0.007$ & $1.377 \pm 0.292$ & $28.918 \pm 0.550$ \\
CY & 0.02 & $0.306 \pm 0.016$ & $0.076 \pm 0.011$ & $0.077 \pm 0.011$ & $1.141 \pm 0.030$ & $28.435 \pm 0.685$ \\
SNFEE & 0.25 & $0.347 \pm 0.059^{*} \Delta$ & $0.103 \pm 0.020$ & $0.104 \pm 0.014^{\Delta}$ & $1.216 \pm 0.073$ & $28.469 \pm 0.803$ \\
SNFWE & 0.25 & $0.275 \pm 0.029^{*}$ & $0.171 \pm 0.221$ & $0.117 \pm 0.013^{\Delta}$ & $1.363 \pm 0.118^{\Delta}$ & $29.652 \pm 0.928^{\Delta}$ \\
\hline
\end{tabular}

*different from the model group, $* p<0.05 ; \Delta$ different from the CY group, ${ }^{\Delta} p<0.05$

TABLE II . INHIBITION OF SNF ON THE GROWTH OF TRANSPLANTED TUMORS IN S180 INDUCED MICE AND ORGAN WEIGHT RATIO $(\mathrm{X} \pm \mathrm{SD})$

\begin{tabular}{cccccc}
\hline Group & $\begin{array}{c}\text { Dosage } \\
(\mathrm{g} / \mathrm{kg})\end{array}$ & $\begin{array}{c}\text { Thymus weight } / \\
\text { Mouse weight } \\
(\mathrm{g} / \mathrm{g})\end{array}$ & $\begin{array}{c}\text { Spleen weight } / \\
\text { Mouse weight } \\
(\mathrm{g} / \mathrm{g})\end{array}$ & $\begin{array}{c}\text { Liver weight } / \\
\text { Mouse weight } \\
(\mathrm{g} / \mathrm{g})\end{array}$ & $\begin{array}{c}\text { Inhibitory rate } \\
(\%)\end{array}$ \\
\hline control & 0 & $0.00356 \pm 0.00020$ & $0.00329 \pm 0.00044$ & $0.04045 \pm 0.00089$ & 0 \\
model & 0 & $0.00363 \pm 0.00036$ & $0.00391 \pm 0.00025$ & $0.04761 \pm 0.00994$ & 48.91 \\
CY & 0.02 & $0.00267 \pm 0.00039$ & $0.00271 \pm 0.00039$ & $0.04014 \pm 0.00110$ & 42.07 \\
SNFEE & 0.25 & $0.00362 \pm 0.00070$ & $0.00366 \pm 0.00052^{\Delta}$ & $0.04274 \pm 0.00289$ & \\
SNFWE & 0.25 & $0.00579 \pm 0.00753$ & $0.00395 \pm 0.00040^{\Delta}$ & $0.04611 \pm 0.00555$ & 54.09
\end{tabular}

*different from the model group, $\mathrm{p}<0.05 ; \Delta$ different from the CY group, $\mathrm{p}<0.05$ 
C. Inhibitory rate on the growth of transplanted tumors in EAC and the organ weight ratio

The anticancer effects of SNF treatment in the EAC-bearing mouse are shown in TABLE III. and TABLE IV. Treatment with CY and SNF resulted in marked suppression of tumor weight. Compared with the model group, the inhibitory rate for the SNFEE and SNFWE groups and the CY group in EAC-bearing mouse were $43.75,59.54$ and $51.15 \%$, respectively. The SNFEE and SNFWE groups showed a significant inhibition of tumor weight compared with the CY-treated group $(\mathrm{P}<$ 0.05). Moreover, the spleen, liver, or thymus index for the SNFEE and SNFWE groups did not decrease significantly compared with the model group [TABLE IV.]. The results indicated that in vivo SNF did not cause any serious toxic effects on the immune systems of the EAC-bearing mice.

TABLE III. ANTITUMOR ACTIVITY OF THE SNF IN MICE TRANSPLANTED EAC TUMOR ( $\quad \mathrm{X} \pm S D$ )

\begin{tabular}{ccccccc}
\hline Group & $\begin{array}{c}\text { Dosage } \\
(\mathrm{g} / \mathrm{kg})\end{array}$ & $\begin{array}{c}\text { Tumor weight } \\
(\mathrm{g})\end{array}$ & $\begin{array}{c}\text { Thymus weight } \\
(\mathrm{g})\end{array}$ & $\begin{array}{c}\text { Spleen weight } \\
(\mathrm{g})\end{array}$ & $\begin{array}{c}\text { Liver weight } \\
(\mathrm{g})\end{array}$ & $\begin{array}{c}\text { Body weight } \\
(\mathrm{g})\end{array}$ \\
\hline control & 0 & $0.000 \pm 0.000$ & $0.107 \pm 0.007$ & $0.096 \pm 0.008$ & $1.249 \pm 0.031$ & $31.087 \pm 0.784$ \\
model & 0 & $0.608 \pm 0.036$ & $0.100 \pm 0.009$ & $0.115 \pm 0.012$ & $1.264 \pm 0.074$ & $29.306 \pm 0.979$ \\
CY & 0.02 & $0.297 \pm 0.027$ & $0.071 \pm 0.014$ & $0.078 \pm 0.011$ & $1.143 \pm 0.035$ & $26.036 \pm 0.800$ \\
SNFEE & 0.25 & $0.342 \pm 0.041^{* \Delta}$ & $0.108 \pm 0.020^{\Delta}$ & $0.096 \pm 0.007^{* \Delta}$ & $1.239 \pm 0.063^{\Delta}$ & $28.076 \pm 0.497^{* \Delta}$ \\
SNFWE & 0.25 & $0.246 \pm 0.016^{* \Delta}$ & $0.095 \pm 0.011^{\Delta}$ & $0.120 \pm 0.014^{\Delta}$ & $1.410 \pm 0.110^{* \Delta}$ & $30.317 \pm 0.513^{* \Delta}$ \\
\hline
\end{tabular}

*different from the model group, $*^{*} p<0.05 ;{ }^{\Delta}$ different from the $\mathrm{CY}$ group, ${ }^{\Delta} p<0.05$

TABLE IV . INHIBITION OF SNF ON THE GROWTH OF TRANSPLANTED TUMORS IN EAC INDUCED MICE AND ORGAN WEIGHT RATIO $(\mathrm{X} \pm \mathrm{SD})$

\begin{tabular}{cccccc}
\hline Group & $\begin{array}{c}\text { Dosage } \\
(\mathrm{g} / \mathrm{kg})\end{array}$ & $\begin{array}{c}\text { Thymus weight } / \\
\text { Mouse weight }(\mathrm{g} / \mathrm{g})\end{array}$ & $\begin{array}{c}\text { Spleen weight } / \\
\text { Mouse weight }(\mathrm{g} / \mathrm{g})\end{array}$ & $\begin{array}{c}\text { Liver weight } / \text { Mouse } \\
\text { weight }(\mathrm{g} / \mathrm{g})\end{array}$ & $\begin{array}{c}\text { Inhibitory rate } \\
(\%)\end{array}$ \\
\hline control & 0 & $0.00344 \pm 0.00020$ & $0.00309 \pm 0.00028$ & $0.04021 \pm 0.00171$ & 0 \\
model & 0 & $0.00341 \pm 0.00028$ & $0.00392 \pm 0.00028$ & $0.04321 \pm 0.00344$ & 0 \\
CY & 0.02 & $0.00273 \pm 0.00057$ & $0.00300 \pm 0.00047$ & $0.04392 \pm 0.00141$ & 51.15 \\
SNFEE & 0.25 & $0.00385 \pm 0.00072^{\Delta}$ & $0.00342 \pm 0.00024^{*}$ & $0.04414 \pm 0.00242$ & 43.75 \\
SNFWE & 0.25 & $0.00313 \pm 0.00033$ & $0.00396 \pm 0.00049^{\Delta}$ & $0.04651 \pm 0.00356$ & 59.54 \\
\hline
\end{tabular}

* different from the model group, $\mathrm{p}<0.05{ }^{{ }^{\wedge}}$ different from the $\mathrm{CY}$ group, $\mathrm{p}<0.05$

D. The effect of SNF treatment on the climbing ability of S180 tumor-bearing mice

As shown in Fig .1, The SNFEE and SNFWE groups improved the climbing time. The effect on the SNFWE group was the greatest and reached significance when compared with the control group $(\mathrm{P}<0.05)$.

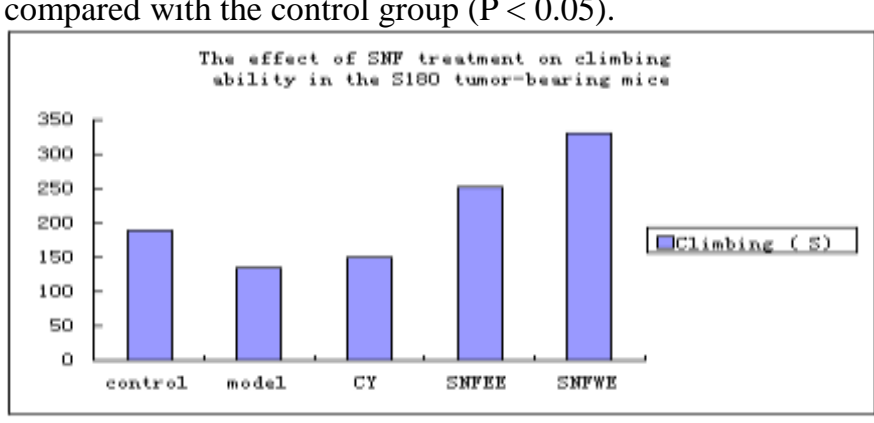

Figure 1. The effect of SNF treatment on climbing ability in the S180 tumor-bearing mice
E. The effect of SNF treatment on the climbing ability of EAC tumor-bearing mice

As shown in Fig .2, when compared with the control group. The SNFEE and SNFWE groups treatment led to an improvement in mice climbing time; however, the effects were most pronounced in the group receiving the SNFWE group $(\mathrm{P}<0.05)$.

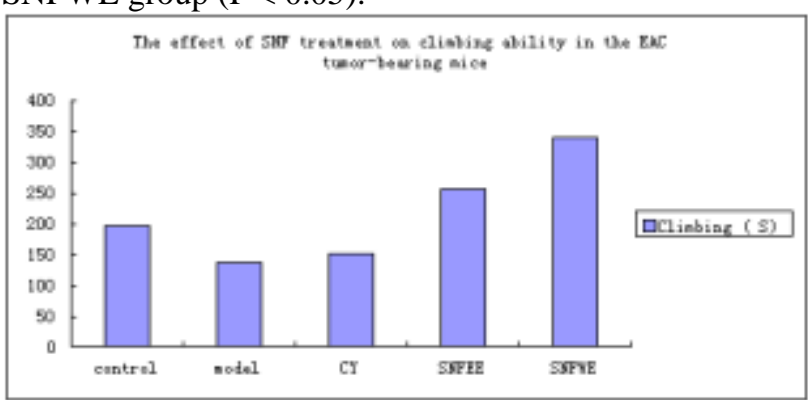

Figure 2. The effect of SNF treatment on climbing ability in the EAC tumor-bearing mice 


\section{F. Blood serum SOD, MDA, and GSH-PX levels in the S180 tumor-bearing mice}

Blood serum analysis revealed that when compared to the SNF -treated mice, in the CY-treated mice, the SOD and GSH-PX content were reduced $(\mathrm{P}<0.05)$. In the SNFWE group, the SOD and GSH-PX serum levels were the closest to control values [Fig .4]. For MDA levels, the SNFWE group was the lowest, when compared with the control group ( $\mathrm{P}<0.05)$ [Fig .3]. Further, when compared against the model group, blood serum SOD and GSH-PX levels in the SNFWE group were significantly reduced (P $<0.05)$. In the SNFWE group, SOD and GSH-PX levels were the highest, being close to or surpassing the control levels.

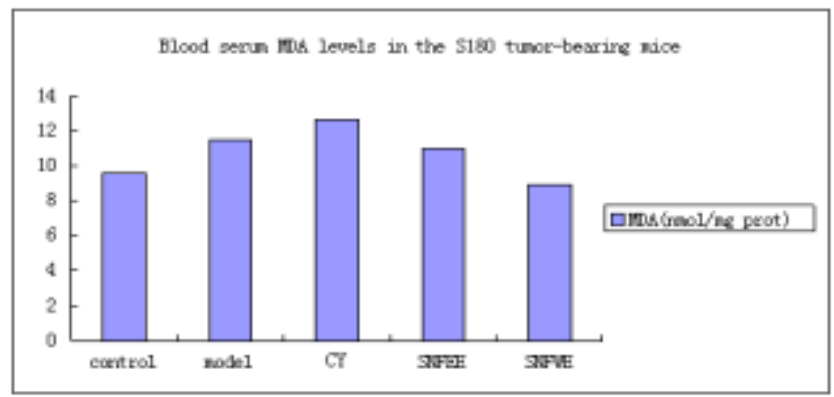

Figure 3. Blood serum MDA levels in the S180 tumor-bearing mice

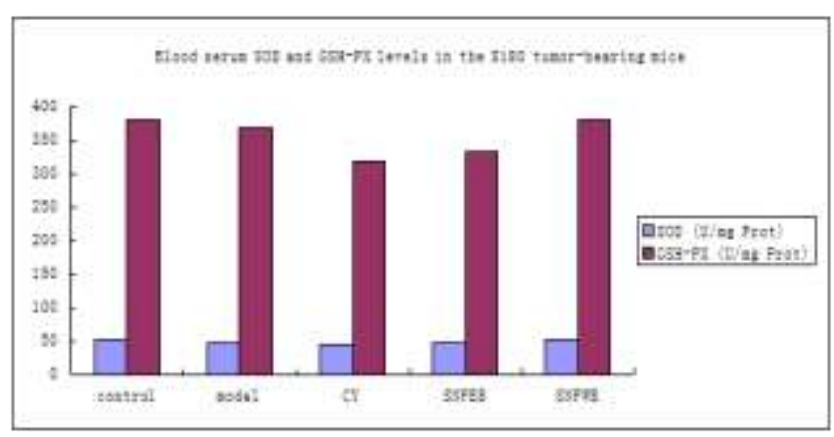

Figure 4. Blood serum SOD and GSH-PX levels in the S180

tumor-bearing mice

\section{G. Blood serum SOD, MDA, and GSH-PX levels in the EAC tumor-bearing mice}

Blood serum analysis revealed that when compared to the SNF -treated mice, in the CY-treated mice, the SOD and GSH-PX content were reduced $(\mathrm{P}<0.05)$. In the SNFWE group, the SOD and GSH-PX serum levels were the closest to control values [Fig .6]. For MDA levels, the SNFWE group was the lowest, when compared with the control group $(\mathrm{P}<0.05)$ [Fig .5]. Further, when compared against the model group, blood serum MDA levels in the SNFWE group were significantly reduced $(\mathrm{P}<0.05)$. In the SNFWE group, blood serum SOD and GSH-PX levels were the highest, being close to or surpassing the control levels.

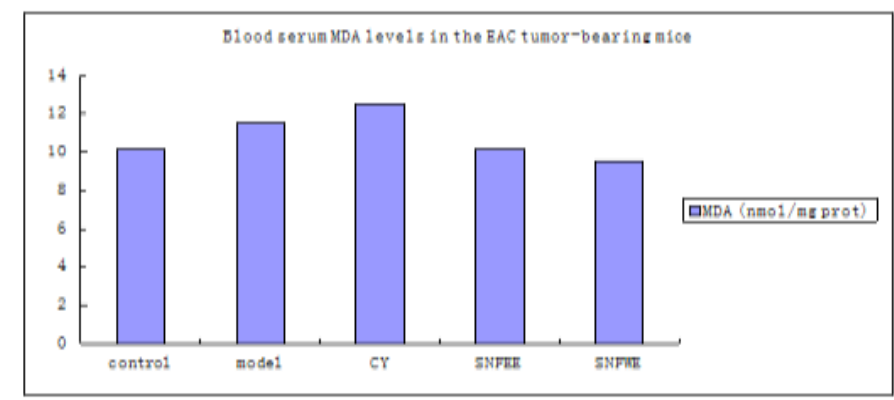

Figure 5. Blood serum MDA levels in the EAC tumor-bearing mice

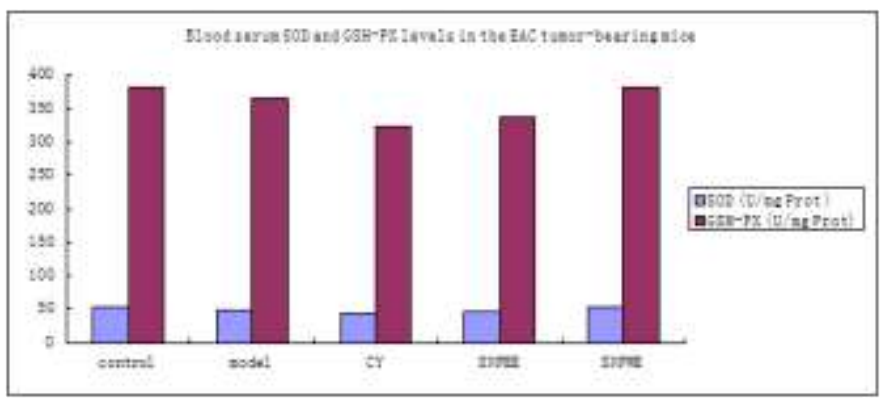

Figure 6. Blood serum SOD and GSH-PX levels in the EAC tumor-bearing mice

\section{H. The effect of SNF-treatment on S180 tumor-bearing mice subjected to hypoxic conditions}

As shown in Fig .7, SNF-treatment can extend the survival time of mice maintained under hypoxic conditions when compared against normoxic conditions. The observed effect was most significant in Ver and SNFWE groups $(\mathrm{P}<0.05)$. The extension rate in the SNFEE, SNFWE groups and the verapamil groups were $20.16,52.05$ and $48.24 \%$, respectively.

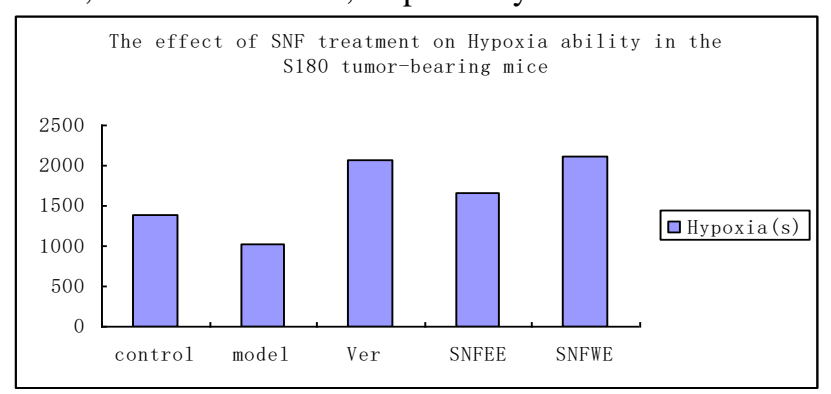

Figure 7. The effect of SNF treatment on Hypoxia ability in the S180 tumor-bearing mice

\section{The effect of SNF-treatment on EAC tumor-bearing mice subjected to hypoxic conditions}

As shown in Fig .8, SNF-treatment can extend the survival time of mice maintained under hypoxic conditions when compared against normoxic conditions, with the effects in the Ver and SNFWE groups being most significant $(\mathrm{P}<0.05)$. The extension rate in the SNFEE, SNFWE groups, and the Vera pamidronate groups were $16.02,41.13$ and $40.62 \%$, respectively. 
The effect of SNF treatment on Hypoxia ability in the EAC

tumor-bearing mice

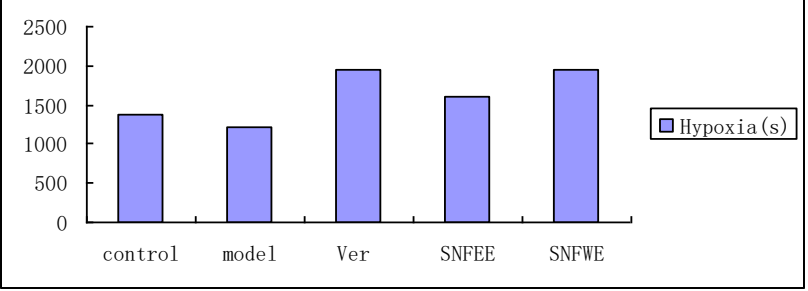

Figure 8. The effect of SNF treatment on Hypoxia ability in the EAC

tumor-bearing mice

\section{DISCUSSION}

We used to study the anti-tumor EAC of SNF made the following main aspects: 1 .We did a study of EAC tumor pathology. 2.Some of the inflammation for the anti-tumor factors were studied,Such as:TNF- $\alpha$, IL-1 $\beta$, IL-2.The results show that the SNFWE against EAC tumor effect [6]. This time we study the S180 and EAC use SNF, and to clarify its anti-tumor mechanisms from different aspects.In this study, the antitumor effects of SNF were evaluated in two mouse xenograft models. The findings of this study show that SNF had potent antitumor activity. Lipid peroxidization is a process that leads to cell damage. Exposure to different chemicals may induce organism to produce the massive free radicals, causing an accumulation of toxicity in the cell and leading to lipid peroxidation damage. SOD is derived from a life of active substances, can eliminate the organism in the new supersedes the old. During hazardous substances. The human body continuously added SOD has anti-aging effect. According to free radical theory, the higher the metabolic rate of an organism, the greater the production of free radicals and the shorter their life span [11-12]. It has been reported that the decrease could be an increase in the glycation of SOD [13-14]. The SNFWE has the obvious opposing effect. MDA is a cell membrane lipid peroxidation product and its plasma contents may mirror the amount of lipid peroxidation. Glutathione peroxidase can be cleared by ROS and $\mathrm{OH}$ induced lipid peroxides, protect the structure and function of cell membrane integrity. Both SOD and GSH-PX can be used to evaluate the oxidation resistance levels as the main biochemistry target. The presence of SOD in the organism can only specifically eliminate the ultra oxygen free radicals, the antioxidase, can the excessive oxygen free radical disproportionate be the hydrogen peroxide, the latter transforms in the catalase and under the GSH-PX catalysis into the water, thus avoids the free radical to cell's harm [15-17]. GSH-PX is also one kind of important antioxidase [18-19]. It may cause the lipid peroxide to return to its original state into fatty acid alcohol, composes the elimination free radical together with SOD the defensive system. MDA is the main production during excessive blood metabolism, SOD and GSH-PX are important antioxidases. The experimental results indicated that the SNFWE has the ability to reduce the oxidative damage in the $\mathrm{S} 180$ and EAC tumor models.

Hypoxia influences the body's energy supply, and will cause ischemia of the heart brain and other important organs, which will soon lead to death [20-22]. Hypoxia refers to oxygen deficiency, hypoxia or lack of oxygen in the air condition. This experiment shows that SNFWE can increase the survival time for the tumor bearing mice living in hypoxic conditions under normal pressure. Moreover, SNFWE can extend the climbing time of these two types of tumor-bearing mice, which indicates that SNFWE has the effect of postponing fatigue. Hypoxia affects the body's energy supply, and ultimately causes critical organs such as the heart and brain to become ischemic leading to necrosis. Our results show that the SNFWE can significantly prolong survival time of tumor-bearing mice under normobaric conditions. The underlying mechanism may be due to SNFWE antioxidant properties that improve the animal's adaptive abilities while under hypoxic conditions. This may consequently aiding in the regulation of various metabolic enzymes, reduced oxygen consumption, free radical scavenging, and increasing the activity of antioxidant enzymes and inhibiting or reducing lipid peroxidation, thereby inhibiting blood rheology degeneration caused by hypoxia. All of these factors may contribute to SNFWE anti-hypoxia effects, but the exact mechanisms remain to be studied in depth. Intragastric administration of SNFWE under a normobaric conditions can significantly prolong mouse survival time during hypoxic conditions. This implies that to some extent SNFWE has protective effects on cardiovascular and cerebral tissue. In short, SNFWE can reduce tumor mass in S180 and EAC tumor bearing mice, inhibit tumor growth, and increase survival time in mice maintained under hypoxic conditions.

The mechanisms leading to these findings may be due to the antioxidant effects of SNFWE leading to an enhanced anti-stress ability, improved adaptation to hypoxic stimuli, improved regulation through adjusting the metabolic enzyme activity, reduced oxygen consumption, enhanced activity of antioxidant enzymes and inhibition or reduction of lipid peroxidation. The net effect of these changes may be to inhibit the changes caused by blood rheology degeneration beacuse of hypoxia and finally an anti-hypoxia effect. However, the mechanism underlying this effect is yet to be fully investigated. In this study, SNFWE was applied with a gavage and treatment with SNFWE increased the survival time for mice maintained under hypoxic conditions (with normal pressure). The study proves that SNFWE may provide protection for the heart and tissues. In conclusion, SNFWE can decrease the tumor in the S180 tumor-bearing group and in the EAC tumor-bearing mice, SNFWE inhibits the growth of tumors, and strengthens the body's immunity and extends the survival time of mice maintained under hypoxic conditions.

ACKNOWLEDGMENT

This research work was supported by Natural Science Foundation of Xinjiang Uighur Autonomous Region, China (No. 2011211B27). 


\section{CONFLICT OF INTEREST}

The authors declare that they have no conflict of interest.

\section{REFERENCES}

[1] A. Zulfiqar, F. Daneel, C. Paulo, A.A. Mitchell, and A.K. Ikhlas, "Nigellidine-4-O-sulfite, the first sulfated indazole-type alkaloid from the seeds of Nigella sativa," J Nat Prod, 1(6):1111-1112, Jun 2008.

[2] Y.M. Liu, J.S. Yang, and Q.H. Liu, "A new alkaloid and its artificial derivative with an indazole ring from Nigella glandulifera," Chem Pharm Bull (Tokyo), 52(4):454-455. Apr 2004.

[3] D. Nguyen, D. Nguyen, H.L. Lyun, H.B. Lee, J.H. Shin, and et al., "Isolation of dioctyl phthalate with high depigmenting effect from Chinese herb Nigella glandulifera Freyn," Journal of Biotechnology, 131(2):S43-S43, 2007.

[4] D.T. Nguyen, D.H. Nguyen, H.L. Lyun, H.B. Lee, J.H. Shin, and et al., "Inhibition of melanogenesis by dioctyl phthalate isolated from Nigella glandulifera Freyn," Journal of microbiology and biotechnology, 2007, 17(10):1585

[5] B. Zhou, X.F. Wei, R.T. Wang, and J.M. Jia, "Quantification of the enzymatic browning and secondary metabolites in the callus culture system of Nigella glandulifera Freyn et Sint," Asian Journal of Traditional Medicines, 2010, (5):3

[6] A. Ainiwaer, X. Xiadiya, F. Chengyu, and M. Dilinuer, "Immunomodulatory and antitumor effects of Nigella glandulifera freyn and sint seeds on ehrlich ascites carcinoma in mouse model," Pharmacognosy Magazine, 2013,9(35): 187-191.

[7] D.D. Zhang, X.Q. Nie, and H.J. Pan, "Study on effect of total saponins from Semen Nigellae on inflammatory mediators and ERK/MAPK pathway in stimulated macrophage," China Journal of Chinese Materia Medica, 2010,19(35):2594-2598.

[8] D.S. Geng, and S.F. Zhang, "The Investigation of Effective Chemical Groups in Uighur Plant Nigella Glandulifera on Relieving Cough, Expelling Phlegm and Anti-inflammatory," Tenth National inflammatory immune Pharmacology Conference Proceedings, 2010,7(29):123-124.

[9] Q. Xiao, X.J. Li, and J. Zhu, "Nigella glandulifera Freyn's antitussive, expectorant, anti-inflammatory effective parts," Proceedings of the Sixth cistanche cum Symposium on Medicinal Plants in the Sand, 2011,5(6):221-227.

[10] X. F. Tan, X.J. LI, and Q. Wang, "HPLC Fingerprint Spectrum of Xinjiang Uyghur Medicine Nigella glandulifera Seed," Journal of Chinese Medicinal Materials, 2011,12(34):1857-1860.
[11] M.E. Inal, G. Kanbak, and E. Sunal, "Antioxidant enzyme activities and malondialdehyde levels related to aging," Clinica chimica acta, 2001, 305(1-2):75-80.

[12] C. Michiels, M. Raes, O. Toussaint, and J. Remacle, "Importance of Se-glutathione peroxidase, catalase, and $\mathrm{Cu} / \mathrm{Zn}$-SOD for cell survival against oxidative stress," Free Radical Biology and Medicine, 1994,17(3):235-248.

[13] P. Huang, L. Feng, A.O. Elizabeth, J.K. Michael, and P. William, "Superoxide dismutase as a target for the selective killing of cancer cells," Nature, 2000, 407(6802):390-395.

[14] A. OkadoMatsumoto, and I. Fridovich, "Subcellular distribution of superoxide dismutases (SOD) in rat liver," Journal of Biological Chemistry, 2001, 276(42):38388.

[15] D. Behne, and W. Wolters, "Distribution of selenium and glutathione peroxidase in the rat," The Journal of nutrition, 1983, 113(2): 456.

[16] M.A. Lovell, W.D. Ehmann, S.M. Butler, and W.R. Markesbery, "Elevated thiobarbituric acid-reactive substances and antioxidant enzyme activity in the brain in Alzheimer's disease," Neurology, 1995, 45(8):1594-1601.

[17] C.D. Thomson, H.M. Rea, V.M. Doesburg, and M.F. Robinson, "Selenium concentrations and glutathione peroxidase activities in whole blood of New Zealand residents," Br J Nutr, 1977, 37(3):457-60

[18] M.P. Look, J.K. Rockstroh, G.S. Rao, K.A. Kreuzer, S. Barton, and et al., "Serum selenium, plasma glutathione (GSH) and erythrocyte glutathione peroxidase (GSH-Px)-levels in asymptomatic versus symptomatic human immunodeficiency virus-1 (HIV-1)-infection," European journal of clinical nutrition, 1997, 51(4):266-272.

[19] S.A. Wohaieb, and D.V. Godin, "Alterations in free radical tissue-defense mechanisms in streptozocin-induced diabetes in rat," Effects of insulin treatment Diabetes, 1987, 36(9):1014-1018

[20] J.N. Barker, "Role of hemoglobin affinity and concentration in determining hypoxia tolerance of mammals during infancy, hypoxia, hyperoxia and irradiation," American Journal of Physiology--Legacy Content, 1957, 189(2):281.

[21] M. Bernaudin, A.S. Nedelec, D. Divoux, E.T. MacKenzie, E. Petit, and P. Schumann-Bard, "Normobaric hypoxia induces tolerance to focal permanent cerebral ischemia in association with an increased expression of hypoxia-inducible factor- 1 and its target genes, erythropoietin and VEGF, in the adult mouse brain," Journal of Cerebral Blood Flow \& Metabolism, 2002, 22(4):393-403.

[22] K. Prass, A. Scharff, K. Ruscher, D. Löwl, C. Muselmann, and et al., "Hypoxia-induced stroke tolerance in the mouse is mediated by erythropoietin," Stroke, 2003, 34(8):1981-1986. 